\title{
Research on process data communication theory in the EMU reconnection UIC gateway and Simulation
}

\author{
Zhang Tong ${ }^{1}$, Li Changxian ${ }^{1}$, Zhao $\mathrm{Ke}^{2}$ \\ ${ }^{1}$ Department of EMU Application and Maintenance Engineering, Dalian Jiaotong University, Dalian, \\ 116028, China \\ ${ }^{2}$ Department of Electronics and Information Engineering, Dalian Jiaotong University, Dalian, \\ 116028, China \\ email: zhang_tong66@126.com
}

Keywords: TCN; UIC; Process Data; PDM

\begin{abstract}
UIC gateway built on UIC556 standard can realize inauguration, process and message data effective configuration and communication between different manufacturers EMUs. With valid configuration of node monitoring database, process data can be properly transferred to the WTB (MVB) bus through filtering and operating of process data marshalling. In this paper, comprehensive and detailed analysis of communication theory and software framework of process data was given within the EMU UIC gateway. In view of the process data transmission routing, marshalling operation procedure and treatment method were studied. By semi-physical simulation platform, the theory above was simulated and functional verified.
\end{abstract}

\section{Introduction}

EMU often need to dynamically grouping or decoding in order to improve operational efficiency and meet the demand of inter-regional and cross-country running. Because our country EMUs adopt many different manufacturers TCN network equipment, different vehicles reconnection or decompilation will inevitably lead to the train network topology changes, at the same time change the original static and dynamic properties, leads to communication barriers after inauguration. Here, TCN standard IEC - 61375 which defined the basic data communication mechanism cannot satisfy the interoperability demands of different manufacturer's vehicles [1]. Equivalent of application layer of TCN standard protocol, UIC556 regards vehicles as the basic unit, defines process and message data frame content, inauguration and a series of related problems [2]. As long as the TCN network equipment of different manufacturers communicate with each other according to the UIC556 standard, use hardware interface consistent with UIC556 communication, or converse interface protocol with UIC gateway simply, then can achieve normal communication of different equipment manufacturers vehicles after interconnection $[3,4]$.

At home and abroad in recent years, more study was focused on MVB and WTB [5], but the theory and design method report on UIC communication and gateway was less. Based on the status of EMU operation in our country, it is an urgent task to master the core technology of interconnection, intercommunication and interoperability of EMUs.

In this paper, the communication mechanism of process data after inauguration in the UIC gateway was researched. First analyzed the interface relationship between UIC gateway and TCN gateway. Then in view of the process data transmission mechanism in UC gateway and communication medium process data marshalling, studied the process data transmission routing and marshalling role and basic algorithms. Finally, the above analysis was simulated and verified on the semi-physical simulation platform. 
The interface relationship between UIC gateway and TCN gateway

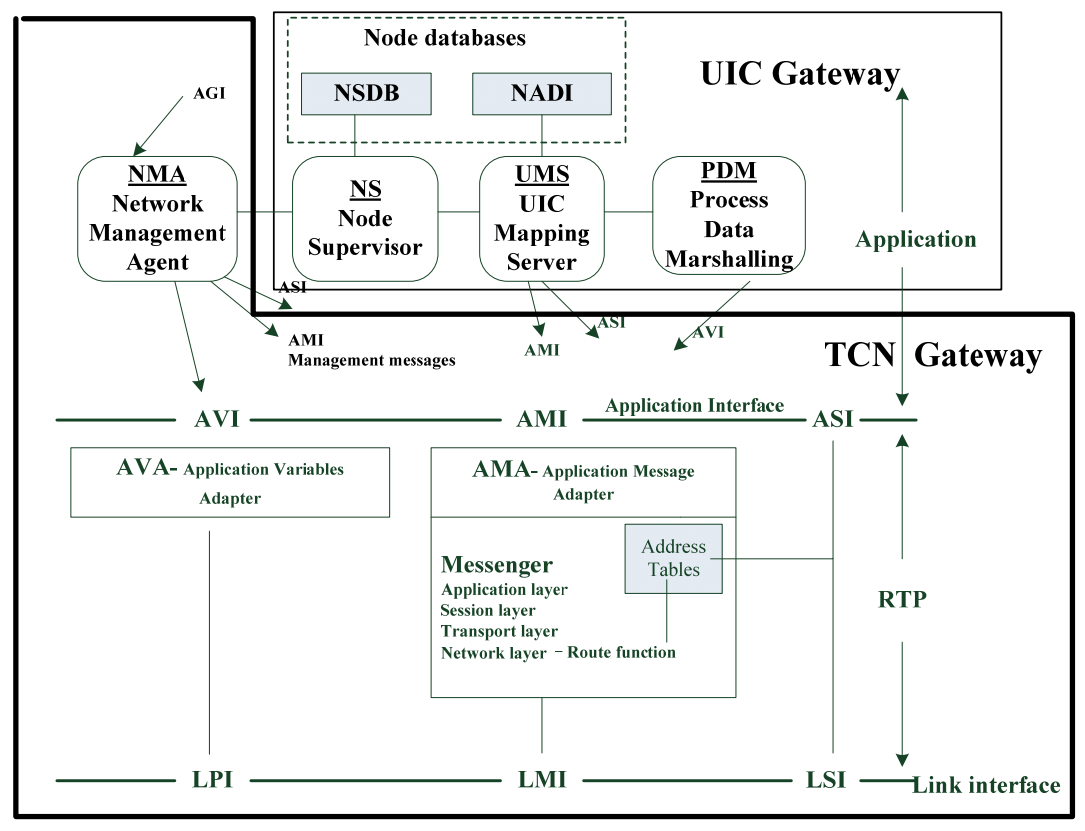

Fig. 1 UIC gateway software architecture

UIC556 laid between UIC application functionality and IEC61375 protocol, is equal to the application layer of TCN protocol. Similarly, UIC gateway and TCN gateway coexistence in an entity, indivisible, interrelated in composition. Application, UIC gateway can fix every process and message data as a frame content which has certain meaning, achieve inauguration and configuration function. TCN protocol stack is under UIC protocol stack software, composed by the WTB and MVB link layer software and real-time protocol, network management software. UIC stack protocol includes node monitoring database (NSDB), UIC mapping server (UMS) and process data marshalling (PDM).

IEC61375 and UIC556 interface is shown in figure 1. UIC556 services is above TCN gateway application layer, and TCN application layer has process data interface (AVI), message data interface (AMI) and superior data interface (ASI). UMS calls and analyzes E message through AMI interface, and decides to call which sub-function to execute operation according to the analyzed message. At the same time, through the ASI interface calls inauguration and other monitoring data, completes the bus management and inauguration, and saves the data into NADI. PDM processes and forwards entry and exit package of process data through AVI interface. NSDB is responsible for inauguration, parameter configuration and control work etc, the corresponding configuration data is saved in NSDB.

\section{Process data transfer mechanism in UIC gateway}

PDM is used for process data transmission between the train bus and vehicle bus. In this process, not all data between MVB and WTB bus must to be transmitted, the gateway needs to filter the data before transmission. On the one hand, when send data from source gateway, filtered data sent from MVB to WTB side, namely export operation mode. On the other hand, when receive data from sink gateway, filtered data sent from WTB to MVB side, namely import operation mode. As for gateway filter which data, how to filter and how to calculation, these all belong to the contents of PDM [6,7].

As shown in figure 2, if process data is the package of export mode, the data first arrives TCN\&UIC gateway MVB LPI interface from MVB physical layer, then up to the gateway AVI interface and is put into PDM for data filtering and processing. After that, the processed data is sent to WTB link layer LPI interface. Eventually, arrives WTB bus physical layer and completes R packet format conversion one time. Vice is called import entry mode package. 


\section{Process data transmission and processing in PDM}

UIC inauguration course can set up communication network topology between different vehicles, and based on this topology PDM becomes a main role to implement network control, it uses WTB train bus to transfer $\mathrm{R}$ packets process data, and executes marshalling treatment to the process data from WTB and MVB.

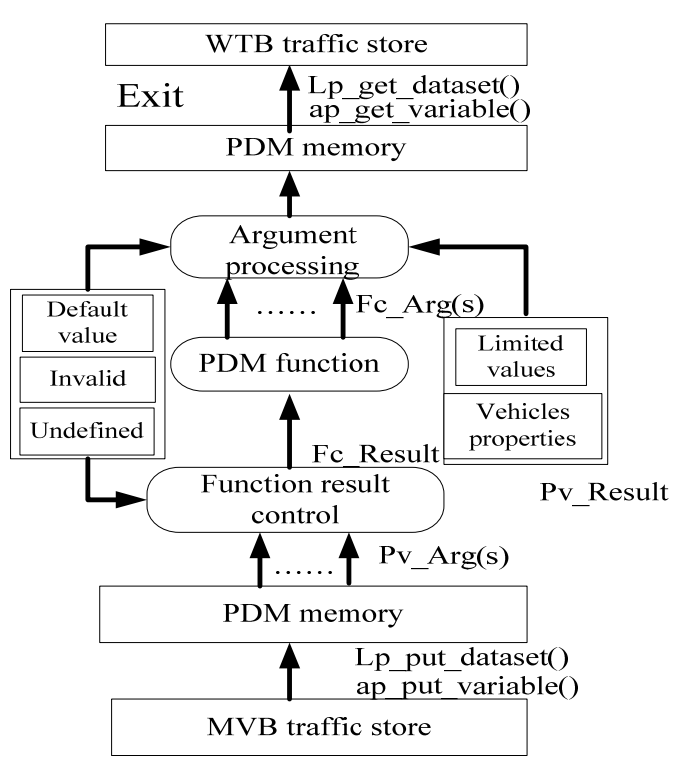

Fig. 2 PDM operating procedures

PDM is classified into output marshalling, input marshalling and internal marshalling. The marshalling which transmits data from MVB traffic store to the source port of WTB traffic store is called output marshalling. The marshalling which transmits data from WTB traffic store to the configured MVB traffic store is called input marshalling. The marshalling which transmission data is between LVR, MVB or LIO port, is belong to vehicle internal transmission and not through WTB bus, so is called internal marshalling. PDM operation is composed by multiple data processing sub modules, used to implement the data acquisition, preprocessing, functional operation and operation result control operation etc, and the operation results will eventually be copied to the target traffic store, as shown in figure 2. If output marshalling, reads data from traffic store port to work buffer, forming parameters Pv_Arg (s). Then, uses argument preprocessing module to preprocess each variable Pv_Arg (s) in PDM memory and get input parameters Fv_Arg (s) of PDM function module. Finally, the results Pv_Result are wrote into traffic store for ready to send.

\section{PDM main steps}

When R telegram entry package arrives, WTB traffic store read all variables to PDM memory, and determine whether is input marshalling. When confirm as input marshalling, check if data's frame type field (FTF) is consistent with NADI's. In the case of FFT correct, execute the marshalling to acquire Pv_Result, otherwise remove the current all WTB data set in PDM memory and all variables becomes invalid. Finally, write the obtained variables from PDM memory to corresponding port of MVB traffic store, shown in Figure 3.

\section{Simulation analysis}

The gateway configuration software is used for configuration and parameters setting core function block such as PDM module, UMS module, WTB/MVB bus manager and traffic store etc. After that, generates the downloadable NSDB file, which contains the whole configuration data of WTB/MVB gateway system, and is used for inauguration, process data and message data communication. 
Process data transmission firstly configures MVB traffic store, such as MVB ports, port length, input data of sink ports, output data of source ports, etc. Secondly, configures WTB traffic store R1, R2 and R3 packet according to UIC556 standard, also can custom packet base on needs in accordance with above three kinds. Then configure vehicle static properties, such as operating railway identification, if vehicle supports group addressing etc. Finally, generates NSDB file for gateway to download.

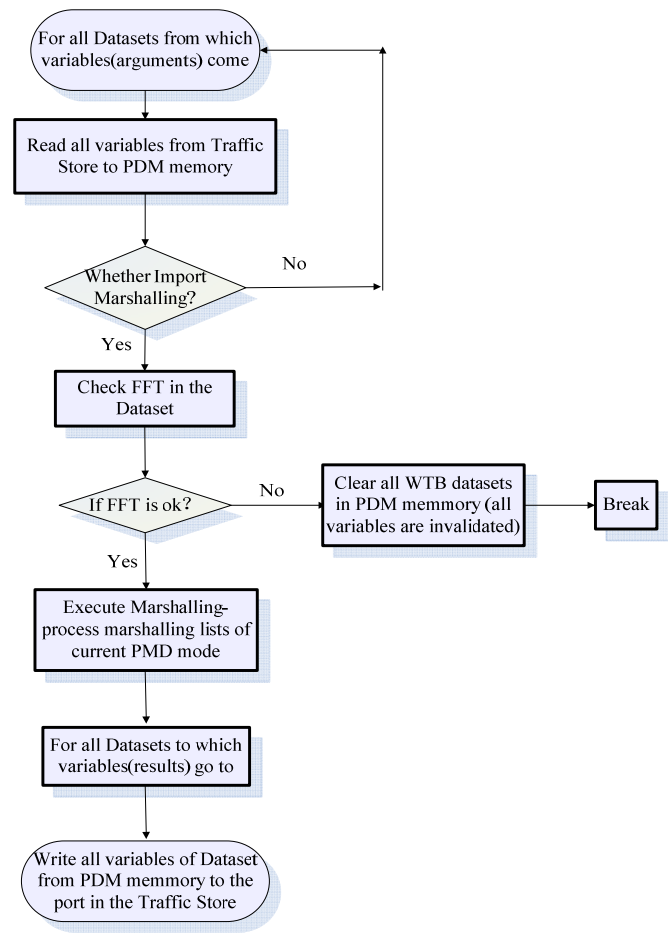

Figure 3. PDM main steps

Semi-physical simulation platform adopted foreign company's TCN \& UIC gateways four set which type was TCN-GW01 / H, 110V power supply, redundant, no I / O interface, for remote shutdown. Additional, equipped with four central processing unit (CCU) and display (HMI) to simulate the corresponding sub-functional units. Use Configuration and Service Station (CSS) software to configure the gateway. Use UIC Train Diagnosis Station (UDS) software to monitor gateway. Each gateway is configured three vehicles, and the platform simulated process data communication respectively on the train.

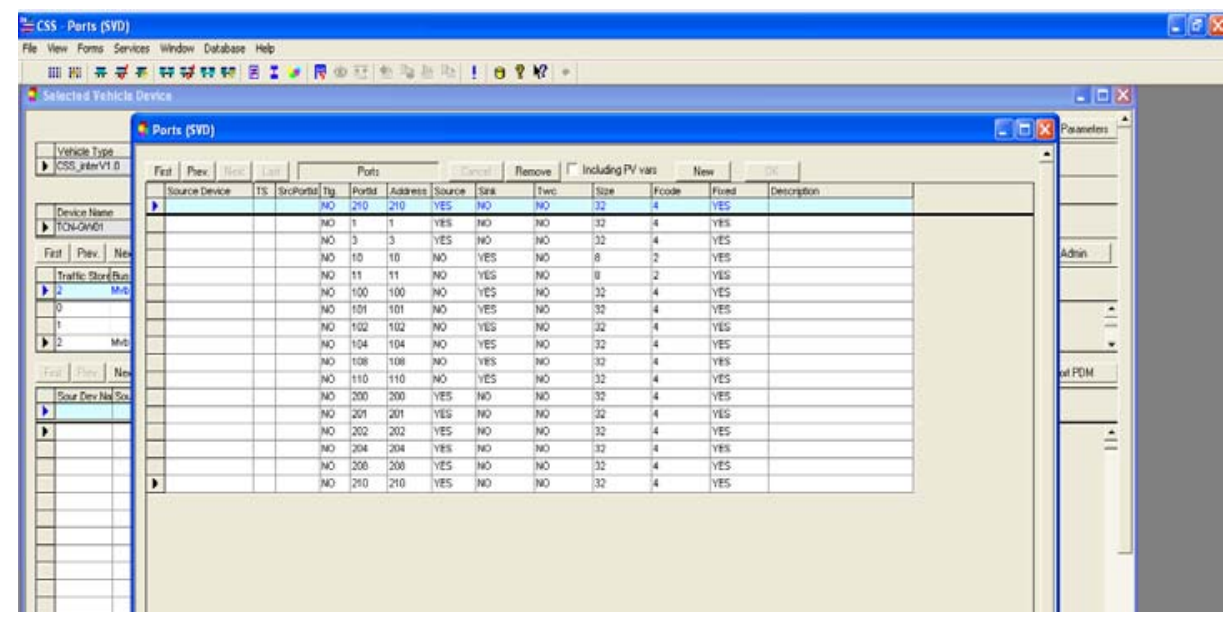

Fig. 4 Sink and source port configuration 

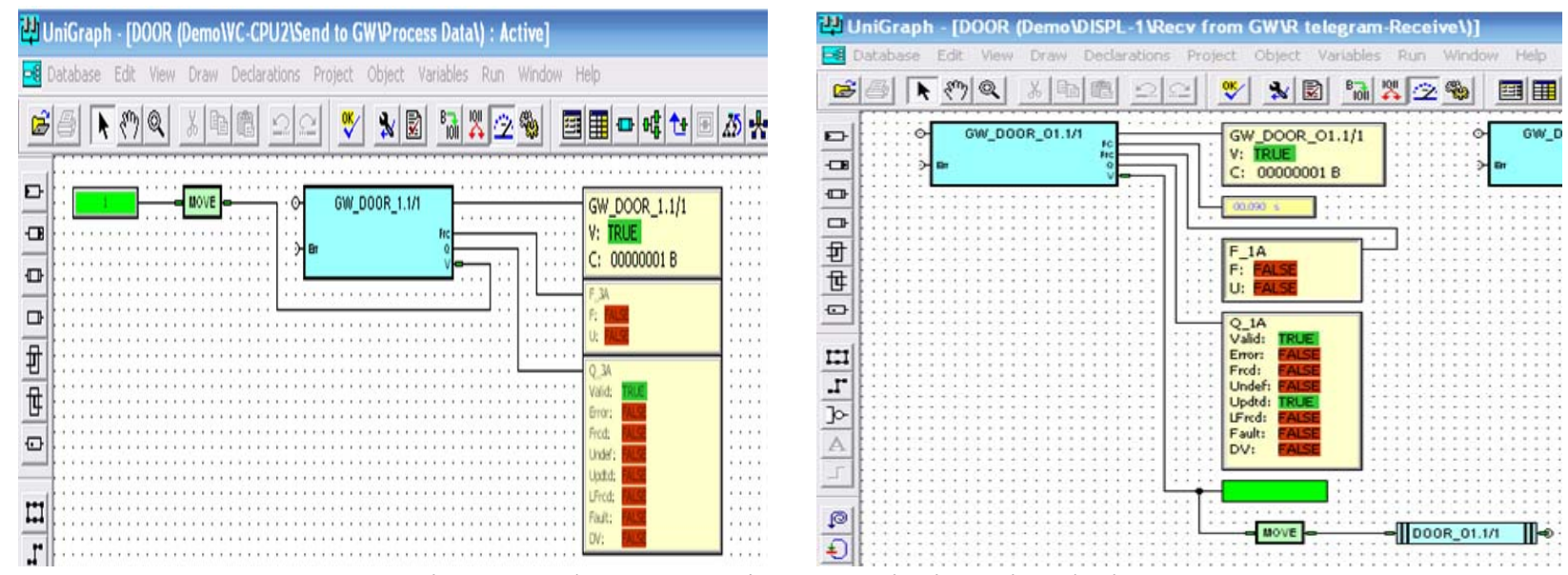

Fig. 5 Telegram 1.1/1 transmission simulations

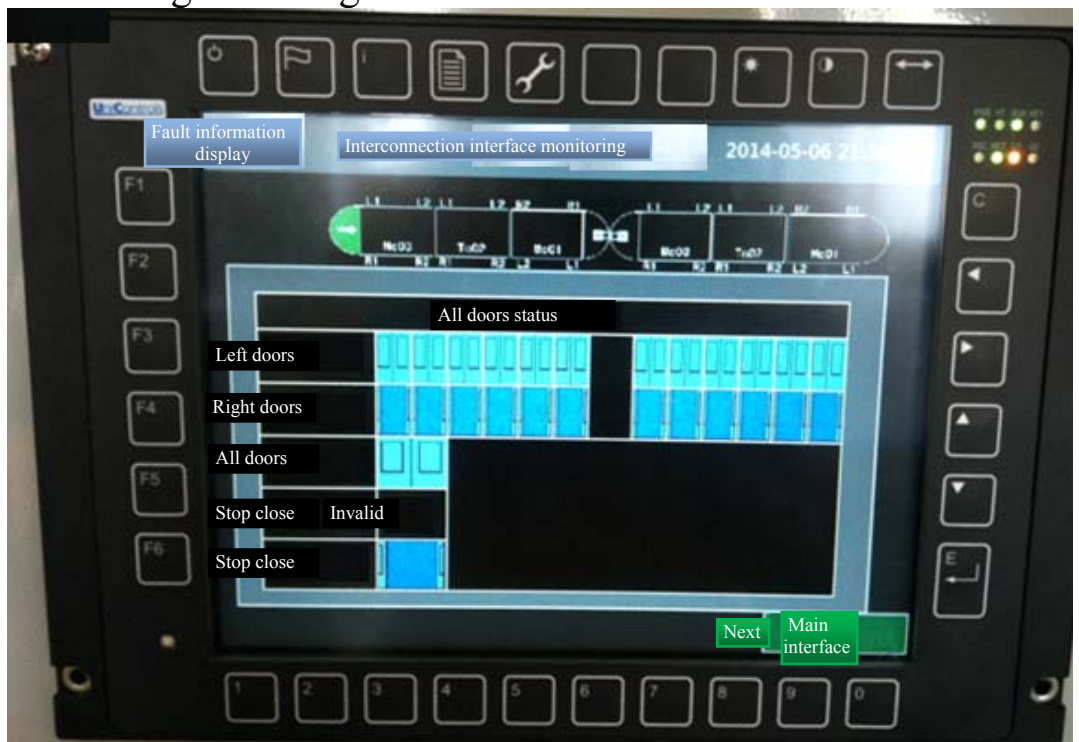

Fig. 6 Doors status display interface on HMI

Process data of train doors R3 telegram 1.1/1 (close all entry doors)was simulated. First, within the CSS, configured sink ports 100 and source ports 200, as shown in figure 4 . Then defined send and receive variables, made variables and defined ports match, defined byte offset and checking variables according to the UIC556 standard R3 packet. Hereafter set up PDM transfer process of process data in gateway, input or output marshalling. Finally, programming with Unicap modular software, as shown in figure 5. All doors status were displayed on Human Machine Interface (HMI), as shown in figure 6 .

\section{Conclusion}

UIC gateway is the key equipment to realize interconnection, intercommunication and interoperability between different models of EMU. UIC and TCN protocol stack software is responsible for it. This paper focused on the transmission mechanism of process data and marshalling operation procedure of PDM in UIC gateway. This study laid the foundation for independent research and development related products, and was conducive to more fully grasp the UIC gateway software technology. Meanwhile, provided a solution for the interconnection, interoperability and interoperability related technology problems of china EMU.

\section{Acknowledgement}

In this paper, the research was sponsored by China Railway Corporation scientific and technological research and development programs (Project No. 2015J007-D). 


\section{References}

[1] Schaefers, C, Hans, G. in: IEC 61375-1 and UIC 556 - international standards for train communication, edited by Vehicular Technology Conference Proceedings. (2002)

[2] UIC556 Leaflet, in: Information Transmission in the train (Train-bus), 3 Edition. (2004)

[3] FEMANDEZ D, JIMENZ J, ANDREU J, in: A TCN Gateway Emulator, edited by Proceedings of IEEE International Symposium on Industrial Electronics, Bilbao: ISIE,. 2911-2916. (2007)

[4] JIMENEZ J, MARTIN J L, BIDARTE U. in: Design of a Master Device for the Multifunction Vehicle Bus, edited by IEEE Transactions on vehicular Technology., 56 (6), 3695-3708. (2007)

[5] JIANG Guotao, in: Design of WTB Controller Based on FPGA, edited by Railway Locomotive \& Car,31 (2), 88-91. (2011)

[6] LI Changxian, LIU Yang, ZHANG Tong. in: Research on UIC Gateway for Interconnection, Intercommunication and interoperability between EMUS, edited by China Railway Science,34 (6), 110-116. (2013)

[7] ZHEN zhen. in: The Design and Implement of PDM Module in UIC Gateway. Dalian University of Technology.(2006) 